\title{
BMJ Open Impact of comprehensive molecular testing to reduce antibiotic use in community-acquired pneumonia (RADICAP): a randomised, controlled, phase IV clinical trial protocol
}

\author{
Gabriela Abelenda-Alonso (10 , ${ }^{1,2}$ Alexander Rombauts, ${ }^{1,3}$ Carlota Gudiol, ${ }^{1,4}$ \\ Yolanda Meije, ${ }^{5}$ Mercedes Clemente, ${ }^{5}$ Lucía Ortega, ${ }^{5}$ Carmen Ardanuy, ${ }^{6}$ \\ Jordi Niubó, ${ }^{6}$ Ariadna Padullés, ${ }^{7}$ Sebastian Videla, ${ }^{8}$ Cristian Tebe, ${ }^{3}$ Jordi Carratalà ${ }^{1,4}$
}

To cite: Abelenda-Alonso G, Rombauts A, Gudiol C, et al. Impact of comprehensive molecular testing to reduce antibiotic use in communityacquired pneumonia (RADICAP): a randomised, controlled, phase IV clinical trial protocol. BMJ Open 2020;10:e038957. doi:10.1136/ bmjopen-2020-038957

- Prepublication history and additional material for this paper are available online. To view these files, please visit the journal online (http://dx.doi org/10.1136/bmjopen-2020038957).

Received 31 March 2020 Revised 11 June 2020 Accepted 15 July 2020

A) Check for updates

(c) Author(s) (or their employer(s)) 2020. Re-use permitted under CC BY-NC. No commercial re-use. See rights and permissions. Published by BMJ.

For numbered affiliations see end of article.

\section{Correspondence to}

Dr Gabriela Abelenda-Alonso; gabi.abelenda.alonso@gmail. com

\section{ABSTRACT}

Introduction Community-acquired pneumonia (CAP) continues to be a major health problem worldwide and is one of the main reasons for prescribing antibiotics. However, the causative agent is often not identified, resulting in antibiotic overtreatment, which is a key driver of antimicrobial resistance and adverse events. We aim to test the hypothesis that comprehensive molecular testing, compared with routine microbiological testing, would be effective in reducing antibiotic use in patients with CAP.

Methods and analysis We will perform a randomised, controlled, open-label clinical trial with two parallel groups (1:1) at two tertiary hospitals between 2020 and 2022. Non-severely immunosuppressed adults hospitalised for CAP will be considered eligible. Patients will be randomly assigned to receive either the experimental diagnosis (comprehensive molecular testing plus routine microbiological testing) or standard diagnosis (only microbiological routine testing). The primary endpoint will be antibiotic consumption measured as days of antibiotic therapy per 1000 patient-days. Secondary endpoints will be de-escalation to narrower antibiotic treatment, time to switch from intravenous to oral antibiotics, days to reaching an aetiological diagnosis, antibiotic-related side effects, length of stay, days to clinical stability, intensive care unit admission, days of mechanical ventilation, hospital readmission up to 30 days after randomisation and death from any cause by 48 hours and 30 days after randomisation. We will need to include 440 subjects to be able to reject the null hypothesis that both groups have equal days of antibiotic therapy per 1000 patient-days with a probability $>0.8$.

Ethics and dissemination Ethical approval has been obtained from the Ethics Committee of Bellvitge Hospital (AC028/19) and from the Spanish Medicines and Medical Devices Agency, and it is valid for all participating centres under existing Spanish legislation. Results will be presented at international meetings and will be made available to patients, their caregivers and funders.

Trial registration number ClinicalTrials: NCT04158492. EudraCT: 2018-004880-29.

\section{Strengths and limitations of this study}

- This study will be the first clinical trial to investigate the impact of comprehensive molecular testing on antibiotic consumption in community-acquired pneumonia (CAP).

- The secondary outcomes will allow us to assess the impact of this stewardship strategy on the safety of the patients admitted with CAP.

- Our findings would help physicians to reduce antimicrobial use when treating patients with CAP.

- The study will be carried out in two centres in the same city in Spain which could be a limitation for the generalisation of the results.

- The molecular testing will not be strictly used as a point-of-care diagnostic method.

\section{BACKGROUND}

Community-acquired pneumonia (CAP) is arguably the most important infectious disease encountered in clinical practice. ${ }^{1}$ In a recent study conducted in the USA, the annual incidence of cases requiring hospitalisation was 24.8 per 10000 adults, with the highest rates recorded in the group aged 65-79years (63.0 per 10000 adults) and in patients aged $\geq 80$ years ( 164.3 per 10000 adults). ${ }^{2}$ A review of 98 studies assessing the burden of CAP among adults in Europe found that its incidence varied by country, age and gender ${ }^{3}$; overall, however, its incidence increases sharply with age.

The prognosis of patients with CAP varies greatly. Among those who do not need hospitalisation, the mortality rate is below $1 \%,{ }^{4}$ but this increases to $4.0 \%-18 \%$ for in-hospital and 30-day mortality for hospitalised patients and up to $50 \%$ for critically ill patients. ${ }^{5}$ The direct and indirect costs of care for patients 
with CAP are also very high. Until now, Streptococcus pneumoniae remains the main causative pathogen of bacterial CAP worldwide. ${ }^{6}$ Nevertheless, only $38.8 \%-66.7 \%$ of CAP episodes achieve microbiological diagnosis using conventional techniques. ${ }^{7-9}$ The growing availability of molecular microbiological tests has been associated with improved identification of respiratory viruses in observational studies of CAP. ${ }^{2}$

In a recent meta-analysis of patients with CAP, $24.5 \%$ had viral disease and mortality was increased in patients with dual bacterial and viral infection. ${ }^{10}$ Broad-spectrum empirical antibiotic coverage in CAP, even in suspected viral CAP, is recommended by current guidelines to cover the most likely aetiologies at presentation. ${ }^{11}$ The same CAP guidelines encourage attempts to broaden, narrow or modify the spectrum of antibiotic therapy based on diagnostic test results, but traditional microbiological investigations have significant limitations and little influence on patient management. ${ }^{12-14}$ Indeed, a randomised trial of 177 patients found that the routine implementation of urine antigen detection tests did not bring any substantial disease or economic-related benefits to patients hospitalised with CAP. ${ }^{15}$ Significantly, CAP is one of the leading causes of antibiotic prescribing, and often, the causative agent is not early identified and patients are overtreated. This overuse of antibiotics is a key driver of antimicrobial resistance and is known to increase the risk of Clostridium difficile infection ${ }^{16}$ and other antibioticrelated adverse events.

Importantly, antibacterial resistance has accelerated at an alarming rate and has led to a global increase in morbidity and mortality. ${ }^{18} 19$ Antimicrobial stewardship is recognised as a key component in the attempts to reduce the associated costs and adverse drug events. ${ }^{20}$ Stewardship strategies include prospective audit with intervention and feedback, clinical pathways, dedicated multidisciplinary teams and the use of biomarkers. In some studies, antimicrobial stewardship strategies have been associated with improvements in antimicrobial use for CAP without negatively affecting clinical outcomes. ${ }^{21-25}$

The current empirical approach to CAP management is largely driven by the fact that no available tests give results fast enough to impact on the decision. It is of special concern since it leads to significant overtreatment with antibiotics and may also cause adverse outcomes due to unexpected pathogens. ${ }^{12}$ Moving to a pathogen-directed approach would therefore help to reduce antibiotic use, improve patient outcomes and lead to a major paradigm shift in current clinical practice guidelines. The development of multiplex real-time PCR assays currently allows for the rapid screening of respiratory specimens, such as nasopharyngeal swabs, for a wide array of respiratory pathogens. ${ }^{24}$ A recent observational study showed that comprehensive molecular testing significantly improved pathogen detection in CAP, particularly in antimicrobialexposed patients. ${ }^{25}$ Interestingly, some authors have evaluated the usefulness of comprehensive molecular testing as a tool for antimicrobial stewardship, although the results have been mixed. ${ }^{26-29}$

\section{Research question and how the question will be addressed}

To date, no randomised trial has evaluated the usefulness of rapid and comprehensive molecular testing as an antibiotic stewardship strategy for CAP with a safety analysis. We therefore want to know, 'Can comprehensive molecular testing reduce antibiotic use in adult patients hospitalised with CAP, without negatively affecting patient outcomes?' To address this question, we will perform a randomised, controlled, open-label clinical trial with two parallel groups. Patients will be randomly assigned to receive comprehensive molecular testing or routine microbiological testing $(1: 1)$.

\section{DESIGN AND METHODS}

\section{Study design and setting}

This randomised, controlled, open-label clinical trial with two parallel groups will be conducted at two tertiary care Spanish hospitals (Bellvitge University Hospital and SCIAS-Barcelona Hospital) between 1 March 2020 and 31 December 2022. It has been registered in the EudraCT and ClinicalTrials databases. Current Good Clinical Practice standards will be followed when performing, monitoring, auditing, analysing and reporting the trial. The protocol follows the Standard Protocol Items: Recommendations for Interventional Trials (SPIRIT) initiative's recommendations, and the study results will be presented in accordance with the Consolidated Standards of Reporting Trials (CONSORT) statement. As required by Spanish legislation, the ethical committee of the principal investigator's institution has approved the study, negating the need for approval from the ethics committee of the other centre.

\section{Patient eligibility and recruitment}

All immunocompetent patients aged 18 years or older and diagnosed with CAP in the emergency department will be screened for study eligibility and recruited consecutively in the two participating hospitals (online supplementary file 1). Participants included in the subsequent randomisation must meet the inclusion criteria and have no reason for exclusion, as indicated below.

\section{Inclusion criteria}

- Adult patients (18 years of age or older) hospitalised with a radiological diagnosis of CAP in the first 24 hours of the admission giving informed consent.

\section{Exclusion criteria}

- Pregnancy or nursing women.

- Severe immunocompromised patients: chemotherapy or radiotherapy in the previous 90 days, use of immunosuppressive drugs, chronic use of corticosteroids at a minimum dose of $15 \mathrm{mg} /$ day in the last 2 weeks, haematopoietic progenitor transplant, solid organ 
transplant and HIV (patient with a CD4 $\leq 200$ cells/ $\left.\mathrm{mm}^{3}\right)$.

- Imminent death (life expectancy $\leq 24$ hours).

- Participation in another clinical trial of pharmacological treatment.

\section{Randomisation}

Randomisation will be performed in computer-generated blocks of 10 by a biomedical statistician from the Biostatistics Unit at Biomedical Research Institute of Bellvitge (IDIBELL). Randomisation will be 1:1 and stratified by hospital site. The random assignment process will be centralised electronically through the electronic data capture itself. Patients will be enrolled and randomly assigned by investigators to receive either the experimental diagnostic approach (comprehensive molecular testing plus to routine microbiological testing) or the standard diagnostic approach (only microbiological routine testing). The allocation list will be stored at the Biostatistics Unit.

\section{Intervention}

Microbiological analysis

The microbiological workup in both study arms will include the following procedures. Blood, pleural fluid and sputum samples will be cultured in standard media. Only good quality sputum samples $(<10$ squamous cell and $>25$ leucocytes per low-power field $(\times 100)$ by Gram stain) will be processed for culture. Urinary S. pneumoniae antigen will be detected by rapid immunochromatographic assay (BinaxNow; Binax), and urinary Legionella pneumophila serogroup 1 antigen will be detected by an immunoenzymatic method (ELISA (Bartels ELISA); Trinity Biotech).

Comprehensive molecular testing in the experimental arm will include the point-of-care molecular detection of respiratory pathogens (FilmArray Pneumonia Panel Plus, BIOFIRE). Semiquantitative detection will be carried out in nasopharyngeal or sputum samples, based on availability from patients, with real-time PCR of 27 microorganisms known to cause pneumonia. Testing will be for the following: S. pneumoniae, Haemophilus influenzae, Moraxella catarrhalis, Pseudomonas aeruginosa, Staphylococcus aureus, Klebsiella pneumoniae, Klebsiella aerogenes, Klebsiella oxytoca, Proteus mirabilis, Serratia marcescens, Streptococcus agalactiae, Streptococcus pyogenes, Enterobacter cloacae, Escherichia coli and Acinetobacter baumannii complex. There will also be a qualitative detection of atypical bacteria (ie, L. pneumophila, Mycoplasma pneumoniae and Chlamydia pneumoniae) and respiratory viruses (ie, influenza virus subtypes $\mathrm{A}$ and $\mathrm{B}$, respiratory syncytial virus, parainfluenza virus, adenovirus, rhinovirus, human metapneumovirus, bocavirus, coronavirus, and Middle East respiratory syndrome coronavirus).

A major limitation of PCR-based tests is to establish whether the detected pathogen is a coloniser. Therefore, we will also perform semiquantification of the relative bacterial abundance. The microbiological findings will be interpreted by an expert microbiologist of the research team and considering the isolated microorganism and clinical context of each case. This information will be provided as soon as possible to the treating physicians via the electronic medical record and a telephone notification.

\section{Antibiotic therapy and follow-up}

Initial empirical antibiotic therapy will be administered in the emergency department in accordance with each hospital's guidelines, which recommend using a $\beta$-lactam agent (eg, ceftriaxone or amoxicillin sodium-clavulanate) with or without a macrolide or fluoroquinolone. Combination therapy is recommended for patients with no initial positive microbiological test. Levofloxacin monotherapy is indicated for Legionella pneumonia and for selected patients, such as those with $\beta$-lactam allergy. The use of immediate targeted antimicrobial treatment, or de-escalation to a narrower spectrum antimicrobial regimen, is recommended if a sensitive pathogen is identified in either study arm.

Attending physicians will determine the duration of antimicrobial therapy. Patients will be seen daily during their hospital stay by attending physicians and by at least one of the investigators. Table 1 describes the procedures and visits that will be carried out by the research team.

\section{Primary endpoint}

Days of antibiotic therapy (DOT) per 1000 patient-day. ${ }^{30}$

\section{Secondary endpoints}

- De-escalation to narrower antibiotic treatment.

- Time to switch from intravenous to oral antibiotics.

- Days to reach an aetiological diagnosis.

- Antibiotic-related side effects (including phlebitis and C. difficile infection).

- Length of hospital stay.

- Days to clinical stability.

- Need for admission to an intensive care unit (ICU).

- Days of mechanical ventilation.

- Need for hospital readmission up to 30 days after randomisation.

- Death from any cause up to 48 hours after randomisation.

- Death from any cause up to 30 days after randomisation.

\section{Follow-up and data collection}

All patients will be assessed daily by a member of the investigating team until discharge or death. A follow-up visit will be arranged for all participating patients, 30 days after discharge. For patients who do not attend follow-up, a structured telephone interview will be used to assess outcomes. A summary of the visit schedule and assessments is displayed in table 1.

Researchers will visit emergency department daily to stimulate patient recruitment. Baseline data will include the following: date and time of randomisation, demographic and epidemiological data, antimicrobials received in the prior 3 months, antimicrobials given as 
Table 1 The procedures that will be carried out at each visit from baseline to the end of the study

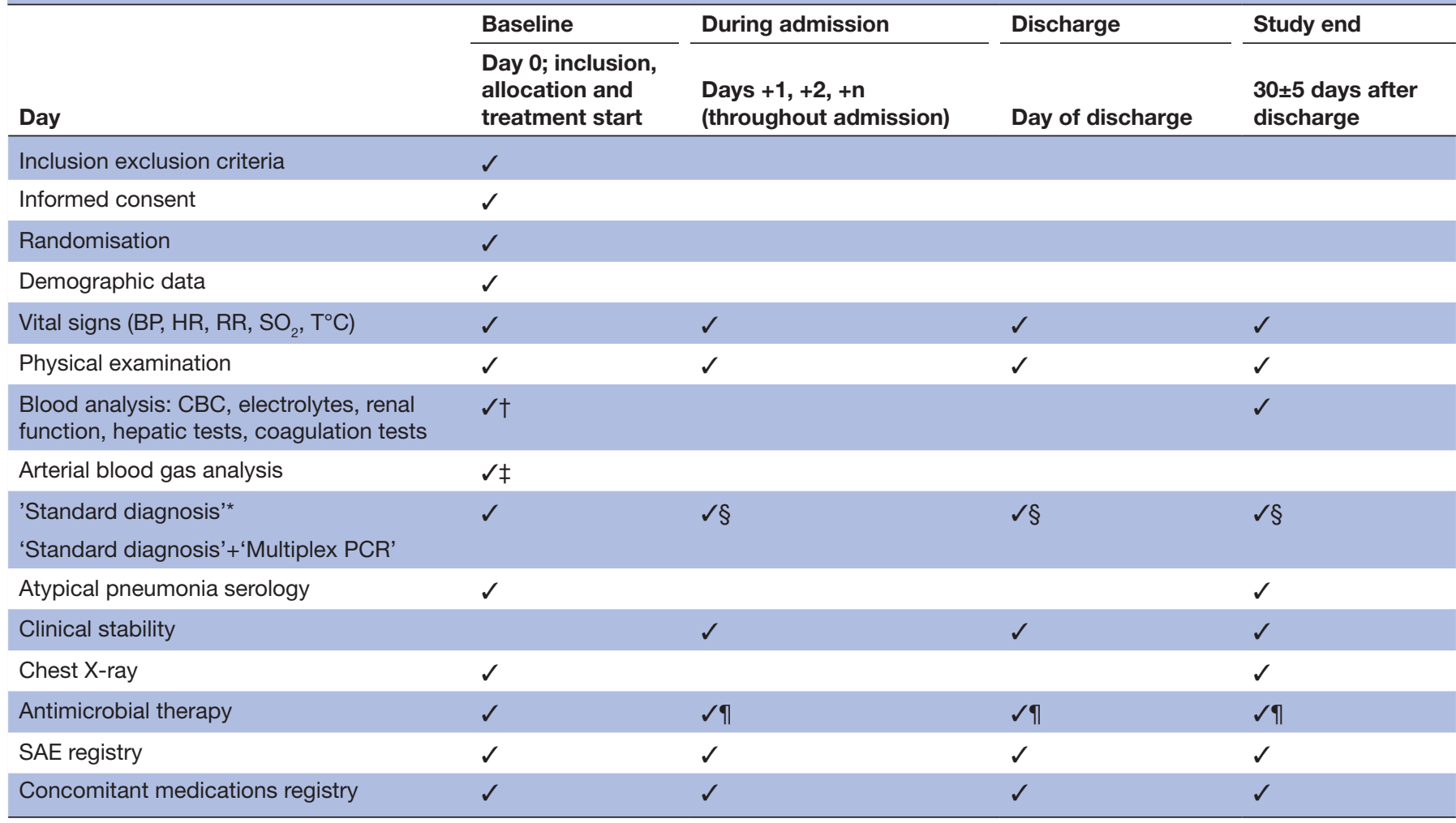

*Standard diagnosis comprised blood culture, sputum culture, urinary antigen test and other cultures at the treating physician's discretion.

†Includes the determination of $\mathrm{C}$ reactive protein.

łlndicated when respiratory insufficiency present.

$\S$ The results of the microbiological tests will be updated daily.

TAll changes in antimicrobial treatment made by treating physicians and the reason for such changes will be collected daily.

$\mathrm{BP}$, blood pressure; $\mathrm{CBC}$, complete blood count; $\mathrm{HR}$, heart rate; RR, respiratory rate; $\mathrm{SAE}$, serious adverse event; $\mathrm{SO}_{2}$, oxygen saturation; $\mathrm{T}^{\circ} \mathrm{C}$, body temperature in degree Celsius.

an outpatient for the current episode, relevant comorbidities, clinical features and variables needed for severity scores. In addition, the dates and times of diagnostic testing and reporting will be collected (ie, for biochemical and microbiological testing), as will those initiating empirical and other antimicrobial treatment. We will also record any reasons for treatment modification. Adverse events related to the study tests and prescribed antibiotics will be collected and communicated to the appropriate authority. Criteria for discontinuing therapy will include patient request and a final diagnosis other than CAP.

All data will be recorded on a secure web application used for building and managing online databases (REDCap). The sponsor and investigator will have access to the final trial dataset. Authorised staff will be free to examine the records for quality assurance and audit purposes.

\section{Patient and public involvement}

This study will not have the participation of patients or the public in the design, recruitment or data interpretation. Nevertheless, the results will be made available to patients, caregivers and funders through press and social media communication.

\section{STATISTICAL ANALYSIS PLAN}

\section{Sample size calculation}

We are planning a clinical trial of a discrete response variable, DOT, in independent control and experimental subjects (1:1). A previous observational study ${ }^{25}$ reported detection rates of $87 \%$ using comprehensive molecular testing and 55\% using 'standard care'. When the aetiology is known, the expected DOT is 8 days, increasing to 11 days when it is unknown. Response within each subject group is non-normally distributed. If the true difference in the experimental and control means is 2 DOTs, we will need to study 220 experimental subjects and 220 control subjects to be able to reject the null hypothesis with a probability above 0.8 . The type I error probability associated with this test of the null hypothesis is 0.05 , assuming an expected dropout rate of $10 \%$.

\section{Type of analysis}

\section{Efficacy analysis}

The baseline characteristics of participants allocated to each study group will be described in tables, using statistics that depend on the data distribution will describe. The primary and secondary endpoints will then be assessed. 
For the primary endpoint, the DOT per 1000 patientdays will be estimated by study group and compared using the Wilcoxon rank-sum test. The probability that an individual from the experimental group presents a lower DOT value than the control group will also be reported.

For the secondary endpoints, Poisson regression modelling will be used to compare the DOT per 1000 patientdays by study group adjusted for age, sex, Charlson Comorbidity Index (CCI) and Pneumonia Severity Index (PSI). The percentages of participants undergoing de-escalation, presenting with antibiotic-related side effects or needing ICU admission will be reported by study group and compared using the $\chi^{2}$ test. Logistic regression modelling will be used to assess de-escalation and antibiotic-related side effects by study group, making adjustments for age, sex, CCI and PSI. Time to switch from intravenous to oral antibiotics, days to reach an etiological diagnosis, length of hospital stay, days to clinical stability, days to de-escalation and days admitted to ICU will be reported by study group and compared using the Wilcoxon rank-sum test. Poisson regression modelling will be used to compare length of hospital stay by study group and compared using the $\chi^{2}$ test. Survival time in the study groups will be analysed by Cox proportional hazards models, adjusting for the factors mentioned above.

All analyses will be performed according to the intention-to-treat principle. We will also conduct a sensitivity analysis in the per-protocol dataset. Statistical significance will be considered where the $p$ value is $\leq 0.05$. Data management and statistical analysis will be performed using statistical package R, V.3.4.3 or greater.

\section{Interim analysis}

To ensure sufficient statistical power, the sample size will be recalculated once half of the initial study population (220 patients) has been recruited. The necessity to increase the initial estimated sample size will be considered based on any encountered variabilities. All data will be monitored by the Data Monitoring Committee of IDIBELL Clinical Research and Clinical Trials Unit, which is cofunded by the European Union and the Instituto de Salud Carlos III and is independent of the sponsor.

\section{Adverse events reporting}

An independent safety monitoring committee will review safety data regarding antimicrobial treatment and incidents regarding comprehensive multiplex PCR, as local legislation requires. All severe adverse events, including related adverse events and death, will be recorded on the electronic case report form according to the Common Terminology Criteria for Adverse Events. The investigators will declare any serious adverse events to the sponsor within 24 hours, and we will present a yearly security update report to the local regulatory agency, consistent with the recommendations of the International Council for Harmonisation.

\section{Indemnities}

This study is classified as a low-intervention trial. According to Spanish legislation (Real Decreto 1090/2015), all damages incurred should be covered by the civil liability insurance of the participating study centres. An ad hoc insurance for the SCIAS-Hospital of Barcelona (private institution) has been taken out.

\section{Trial status}

Recruitment started on 24 February 2020 and trial enrolment is planned to run from February 2020 to December 2022. The present manuscript describes the protocol authorised at submission: version 3.0;8 October 2019.

\section{Protocol amendments}

Any protocol modifications will not become effective until approved by relevant authorities and by the Drug Research Ethics Committee (CEIm). Exceptions are changes to protect patients from imminent harm and those concerning exclusively logistic or administrative aspects.

A protocol amendment adding pneumonia caused by SARS-CoV-2 to the exclusion criteria has been submitted and is currently pending decision from the relevant authorities and the Drug Research Ethics Committee.

\section{ETHICS AND DISSEMINATION}

Ethical approval was obtained by the Clinical Research Ethics Committee of Bellvitge (reference number HUBINF-RADICAP; AC028/19) and the Spanish Medicines and Medical Devices Agency (AEMPS) and is valid for all participating centres that were located within the same autonomous community. This study did not have the participation of patients or the public in its design. Informed consent will be obtained from all patients or their relatives by the principal investigators. The study will be carried out in accordance with current Spanish (Real Decreto 1090/2015) and European (Regulation $536 / 2014$ ) legislation, and it will follow the principles of the Declaration of Helsinki (ISO 14155:2011).

We will communicate preliminary results to international and national infectious disease congresses and will publish a manuscript with the final study results in an appropriate peer-reviewed journal. Any formal presentation or publication of data collected from this study will be considered as a joint publication by the participating investigators and will follow the recommendations of the International Committee of Medical Journal Editors (ICMJE). Furthermore, the results of this will be made available to patients, caregivers and funders. Individual participant data that underlie the results of the study will be available on request after deidentification. To gain access, data requestors will need to sign a data access agreement. 


\section{DISCUSSION}

Although a large number of causative pathogens are associated with CAP, the aetiology remains unknown in around $40 \%-50 \%$ of cases. ${ }^{1}$ In addition, antimicrobial resistance is a critical public health issue that has led to the need for antimicrobial stewardship strategies worldwide. When managing CAP, these strategies seek to promote antimicrobial de-escalation, to reduce the treatment duration and to ensure switching to oral treatment whenever possible.

Some studies have shown that comprehensive molecular testing could reduce antibiotic use in CAP, although with some drawbacks. For example, Gelfer et al conducted a non-blinded cluster randomisation trial of 59 patients with viral CAP and showed that the use of multiplex PCR could reduce duration (days) of antimicrobial therapy $(\mathrm{p}=0.003) .{ }^{26}$ However, no safety analysis was performed to check outcomes and the authors found no differences between patients with only bacterial CAP and those coinfected with both viral and bacterial CAP. In a larger quasi-randomised study of 545 patients with lower respiratory tract infection, Andrews et al showed that there was no association between multiplex PCR use and either reduced antimicrobial treatment or reduced hospital stays. ${ }^{31}$ Nevertheless, this work was limited by using a heterogeneous sample of patients with lower respiratory tract infection and by failing to conduct a safety analysis. Furthermore, in a recent randomised controlled study of 800 patients with lower respiratory tract infection, ${ }^{29}$ researchers found that multiplex PCR use was associated with a reduced duration of intravenous antibiotic use, a shorter length of hospital stay and lower costs of hospitalisation. Major limitations of this study were that it was a single centre study, and as with the other studies, ${ }^{26} 2931$ it failed to include a safety analysis. As suggested by Vos and Oosterheert, ${ }^{32}$ safety analysis is critical when we seek to assess the real outcomes and costs associated with the implementation of new diagnostic methods for antimicrobial stewardship.

We hypothesise that adding comprehensive molecular tests to the routine microbiological diagnostic process could help to reduce antibiotic consumption without negatively affecting morbidity and mortality. Indeed, it may even help to reduce the negative effects associated with antimicrobial therapy, such as phlebitis and C. difficile infection. Only by including a safety analysis can we assess these aspects. This randomised controlled trial is designed to test this hypothesis, and we anticipate that the results will uncover the real-world clinical impact of this techniques because the research team will not interfere with decisions about treatment adjustment. Even negative results will add to our knowledge about the role of this technique in clinical settings, specifically regarding any safety issues that arise from its implementation in usual clinical practice. In conclusion, we expect that our findings will help doctors to improve antimicrobial stewardship by safely reducing the use of broad-spectrum antimicrobials in patients with CAP.
Author affiliations

${ }^{1}$ Infectious Diseases, Bellvitge University Hospital, L'Hospitalet de Llobregat, Barcelona, Spain

${ }^{2}$ Bellvitge Institute for Biomedical Research, Barcelona, Spain

${ }^{3}$ Statistics Advisory Service, Institut dl'Investigacio Biomedica de Bellvitge, L'Hospitalet de Llobregat, Spain

${ }^{4}$ University of Barcelona, Barcelona, Catalunya, Spain

${ }^{5}$ Infectious Diseases Unit-Department of Internal Medicine, Hospital de Barcelona, Barcelona, Catalunya, Spain

${ }^{6}$ Department of Clinical Microbiology Unit, Bellvitge University Hospital, L'Hospitalet de Llobregat, Barcelona, Spain

${ }^{7}$ Department of Farmacology, Bellvitge University Hospital, L'Hospitalet de Llobregat, Barcelona, Spain

${ }^{8}$ Department of Clinical Farmacology, Bellvitge University Hospital, L'Hospitalet de Llobregat, Barcelona, Spain

\section{Twitter Gabriela Abelenda-Alonso @gabelenda1}

Acknowledgements This study will be supported by Plan Nacional de I+D+ i 2013-2016 and Instituto de Salud Carlos III, Subdirección General de Redes y Centros de Investigación Cooperativa, Ministerio de Economía, Industria y Competitividad, Spanish Network for Research in Infectious Diseases (REIPI RD16/0016/0008; RD16/0016/0008) and cofinanced by European Development Regional Fund "A way to achieve Europe", Operative programme Intelligent Growth 2014-2020. We thank CERCA Programme/Generalitat de Catalunya for institutional support.

Contributors JC, GAA, CG, CA, AP, CT and SV contributed to the concept and design of the trial. The inclusion, data collection and interpretation will be performed by JC, GAA, AR, YM, CG, LO, MC, CA and JN. CT developed the statistical analysis plan and calculated the sample size. JC and GAA participated in the writing and submission for publication. All authors have read and approved the manuscript.

Funding This study is supported by a grant from the 201808-10 project, funded by La Marató de TV3.

Competing interests None declared.

Patient consent for publication Not required.

Provenance and peer review Not commissioned; externally peer reviewed.

Open access This is an open access article distributed in accordance with the Creative Commons Attribution Non Commercial (CC BY-NC 4.0) license, which permits others to distribute, remix, adapt, build upon this work non-commercially, and license their derivative works on different terms, provided the original work is properly cited, appropriate credit is given, any changes made indicated, and the use is non-commercial. See: http://creativecommons.org/licenses/by-nc/4.0/.

ORCID iD

Gabriela Abelenda-Alonso http://orcid.org/0000-0001-8158-9934

\section{REFERENCES}

1 Bartlett JG. Diagnostic tests for agents of community-acquired pneumonia. Clin Infect Dis 2011;52:S296-304.

2 Jain S, Self WH, Wunderink RG, et al. Community-acquired pneumonia requiring hospitalization among U.S. adults. $N$ Engl J Med 2015;373:415-27.

3 Welte T, Torres A, Nathwani D. Clinical and economic burden of community-acquired pneumonia among adults in Europe. Thorax 2012;67:71-9.

4 Carratalà J, Fernández-Sabé N, Ortega L, et al. Outpatient care compared with hospitalization for community-acquired pneumonia: a randomized trial in low-risk patients. Ann Intern Med 2005;142:165-72.

5 Prina E, Ranzani OT, Torres A. Community-acquired pneumonia. Lancet 2015;386:1097-108.

6 Wunderink RG, Waterer GW. Clinical practice. community-acquired pneumonia. N Engl J Med 2014;370:543-51.

7 Bjarnason A, Westin J, Lindh M, et al. Incidence, etiology, and outcomes of community-acquired pneumonia: a population-based study.. Open Forum Infect Dis 2018;5:ofy010.

8 Shibli F, Chazan B, Nitzan O, et al. Etiology of community-acquired pneumonia in hospitalized patients in northern Israel. Isr Med Assoc J 2010;12:477-82. 
9 Musher DM, Roig IL, Cazares G, et al. Can an etiologic agent be identified in adults who are hospitalized for community-acquired pneumonia: results of a one-year study. J Infect 2013;67:11-18.

10 Burk M, El-Kersh K, Saad M, et al. Viral infection in communityacquired pneumonia: a systematic review and meta-analysis. Eur Respir Rev 2016;25:178-88.

11 Metlay JP, Waterer GW, Long AC, et al. Diagnosis and treatment of adults with community-acquired pneumonia. An official clinical practice guideline of the American thoracic Society and infectious diseases Society of America. Am J Respir Crit Care Med 2019;200:e45-67.

12 Basnayake TL, Waterer GW. Rapid diagnostic tests for defining the cause of community-acquired pneumonia. Curr Opin Infect Dis 2015;28:185-92.

13 Falguera M, Trujillano J, Caro S, et al. A prediction rule for estimating the risk of bacteremia in patients with community-acquired pneumonia. Clin Infect Dis 2009;49:409-16.

14 Shoji H, Domenech A, Simonetti AF, et al. The Alere BinaxNOW pneumococcal urinary antigen test: diagnostic sensitivity for adult pneumococcal pneumonia and relationship to specific serotypes. $J$ Clin Microbiol 2018;56:e00787-17.

15 Falguera M, Ruiz-González A, Schoenenberger JA, et al. Prospective, randomised study to compare empirical treatment versus targeted treatment on the basis of the urine antigen results in hospitalised patients with community-acquired pneumonia. Thorax 2010;65:101-6.

16 Stevens V, Dumyati G, Fine LS, et al. Cumulative antibiotic exposures over time and the risk of Clostridium difficile infection. Clin Infect Dis 2011;53:42-8.

17 Chalmers JD, Akram AR, Singanayagam A, et al. Risk factors for Clostridium difficile infection in hospitalized patients with communityacquired pneumonia. J Infect 2016;73:45-53.

18 Laxminarayan R, Duse A, Wattal C, et al. Antibiotic resistance-the need for global solutions. Lancet Infect Dis 2013;13:1057-98.

19 Ammerlaan HSM, Harbarth S, Buiting AGM, et al. Secular trends in nosocomial bloodstream infections: antibiotic-resistant bacteria increase the total burden of infection. Clin Infect Dis 2013;56:798-805.

20 Schuts EC, Hulscher MEJL, Mouton JW, Natsch S, Hertogh C, Wolfs TFW, et al. Current evidence on hospital antimicrobial stewardship objectives: a systematic review and meta-analysis. Lancet Infect Dis 2016;16:847-56.
21 Viasus D, Vecino-Moreno M, De La Hoz JM, et al. Antibiotic stewardship in community-acquired pneumonia. Expert Rev Anti Infect Ther 2017;15:351-9.

22 Carratalà J, Garcia-Vidal C, Ortega L, et al. Effect of a 3-step critical pathway to reduce duration of intravenous antibiotic therapy and length of stay in community-acquired pneumonia: a randomized controlled trial. Arch Intern Med 2012;172:922-8.

23 Kaziani K, Sotiriou A, Dimopoulos G. Duration of pneumonia therapy and the role of biomarkers. Curr Opin Infect Dis 2017;30:221-5.

24 Brendish NJ, Schiff HF, Clark TW. Point-of-care testing for respiratory viruses in adults: the current landscape and future potential. J Infect 2015;71:501-10.

25 Gadsby NJ, Russell CD, McHugh MP, et al. Comprehensive molecular testing for respiratory pathogens in community-acquired pneumonia. Clin Infect Dis 2016;62:817-23.

26 Gelfer G, Leggett J, Myers J, et al. The clinical impact of the detection of potential etiologic pathogens of community-acquired pneumonia. Diagn Microbiol Infect Dis 2015;83:400-6.

27 Andrews D, Chetty Y, Cooper BS, et al. Multiplex PCR point of care testing versus routine, laboratory-based testing in the treatment of adults with respiratory tract infections: a quasi-randomised study assessing impact on length of stay and antimicrobial use. BMC Infect Dis 2017;17:671.

28 Roisin S, Huang T-D, de Mendonça R, et al. Prospective evaluation of a high multiplexing real-time polymerase chain reaction array for the rapid identification and characterization of bacteria causative of nosocomial pneumonia from clinical specimens: a proof-of-concept study. Eur J Clin Microbiol Infect Dis 2018;37:109-16.

29 Shengchen D, Gu X, Fan G, et al. Evaluation of a molecular point-ofcare testing for viral and atypical pathogens on intravenous antibiotic duration in hospitalized adults with lower respiratory tract infection: a randomized clinical trial. Clin Microbiol Infect 2019;25:1415-21.

30 Clinical Document Architecture (CDA). Antimicrobial use and resistance (AUR) module. Available: https://www.cdc.gov/nhsn/pdfs/ pscmanual/11pscaurcurrent.pdf [Accessed 30 Mar 2020].

31 Andrews D, Chetty Y, Cooper BS, et al. Multiplex PCR point of care testing versus routine, laboratory-based testing in the treatment of adults with respiratory tract infections: a quasi-randomised study assessing impact on length of stay and antimicrobial use. BMC Infect Dis 2017;17:10:671.

32 Vos LM, Oosterheert JJ. Testing for viral infections in severe lower respiratory tract infections; the unpredictable effects of diagnostic certainty. Clin Microbiol Infect 2019;25:1302-3. 